\title{
Searching for Supersymmetry with the ATLAS Detector at the LHC
}

\author{
Bruce A. Schumm \\ On behalf of the ATLAS Collaboration \\ Santa Cruz Institute for Particle Physics and the University of California at Santa Cruz, 1156 High \\ Street, Santa Cruz, CA, 95064, USA
}

\begin{abstract}
Highlights of ATLAS searches for signatures associated with Supersymmetry are presented. The analyses presented make use of between 1.0 and $4.7 \mathrm{fb}^{-1}$ of LHC data at $\sqrt{s}=7 \mathrm{TeV}$.
\end{abstract}

Keywords: Supersymmetry, Searches

PACS: 12.60.Jv, 13.85.Rm, 14.80.Ly, 14.80.Nb

Supersymmetry (SUSY) introduces a symmetry between fermions and bosons, resulting in a SUSY partner (sparticle) with identical quantum numbers except for a difference of a half-unit of spin from each corresponding Standard Model (SM) particle. The introduction of the symmetry provides a compelling mechanism for the stabilization of the Higgs mass in Grand Unified Theory (GUT) scenarios. To avoid the violation of lepton and baryon number conservation, a multiplicative quantum number $R$ can be introduced such that $R_{S M}=+1$ and $R_{S U S Y}=-1$. In turn, ' $R$-parity' conservation demands that the lightest sparticle (LSP) be stable. Should this LSP be weakly-interacting, this leads to a dark matter candidate and allows for the use of missing transverse energy $E_{\mathrm{T}}^{\text {miss }}$ as a signature for the discovery of SUSY states.

Since sparticle states have yet to be observed, SUSY must be a broken symmetry. Several schemes have been put forward to engineer the breaking of SUSY, including 'supergravity' (SUGRA) [1], 'gauge mediation' (GMSB) [2], and 'anomaly mediation' (AMSB) [3]. Each of these has a predominant phenomenology that, taken together, drives most of the interpretation of the results of searches for supersymmetric phenomena. SUGRA models tend to feature a generic 'neutralino' LSP $\left(\tilde{\chi}_{1}^{0}\right)$ composed of an admixture of the bino, neutral wino, and higgsino states. GMSB models feature a light $(<1 \mathrm{GeV})$ gravitino LSP, and a stau or $\tilde{\chi}_{1}^{0}$ next-to-lightest sparticle (NLSP); in the latter case, the makeup of the $\tilde{\chi}_{1}^{0}$ directly impacts the experimental signature that is explored to probe the model. AMSB models tend to feature a wino-like LSP, with near-degeneracy between the lightest neutralino state that forms the LSP and the lightest chargino state $\left(\tilde{\chi}_{1}^{+}\right)$.

Most of the models used to inspire searches and interpret their results derive from these three SUSY breaking scenarios, and fall into two major classes: 'minimal' or 'constrained' models and 'phenomenological' models. For the former, unification at the GUT scale, as well as its evolution to the accessible electroweak (EW) scale, is determined by a minimal numbers of parameters (five for both the cases of minimal SUGRA (mSUGRA/CMSSM) and GMSB (mGMSB)). Phenomenological models, on 
the other hand, focus on the characteristic phenomenological content of the underlying SUSY-breaking scheme, while decoupling all other sparticle states and allowing the masses of the accessible states to vary free of the interrelations imposed by the physics of the constrained models.

A number of ATLAS SUSY results make use of the full data sample from the $\sqrt{s}=7$ $\mathrm{TeV} 2011$ dataset. For an integrated luminosity of this magnitude, accessible coloredsparticle masses are produced by rare collisions from the parton distribution function (PDF) tails, and thus occupy a region for which the cross section falls very steeply with sparticle mass. Since it is thus somewhat difficult to extend the mass reach with additional luminosity, much current effort is aimed at closing gaps in the coverage of SUSY parameter space. This includes the use of novel analysis and triggering techniques, as well as placing more direct focus on models for which all strongly-coupled sparticles are decoupled and SUSY production proceeds only through EW diagrams.

A general characteristic of SUGRA-like phenomenology is the production of a pair of colored sparticles (squarks and gluinos), each of which undergoes a subsequent decay, emitting several colored SM particles, to the LSP. This process tends to produce events with a number of jets and significant $E_{\mathrm{T}}^{\text {miss }}$. To avoid overlap with analyses that focus on lepton production, events with identified leptons are rejected. Six complementary channels (labeled $A-E, A^{\prime}$ in Fig. 1) are defined, based upon the number of jets and the required magnitude $E_{\mathrm{T}}^{\text {miss }} / m_{\text {eff }}$ of the missing-transverse-energy significance. Requirements are also placed directly on the transverse mass $m_{\text {eff }}$, defined to be the sum of the transverse energy of the reconstructed jets plus $E_{\mathrm{T}}^{\mathrm{miss}}$, which must be large for strongly produced states at the mass scales under study. For each point in the parameter space of the model under study, the channel with the best expected sensitivity is chosen for discovering or excluding the model. No significant excess is observed in any channel; Fig. 1 shows the expected and observed exclusion limits, for an integrated luminosity of $4.7 \mathrm{fb}^{-1}$, in the plane of universal GUT-scale boson $\left(m_{0}\right)$ and fermion $\left(m_{1 / 2}\right)$ mass in the highly-constrained mSUGRA/CMSSM model [4]. Contours of the squark and gluino mass are also shown; limits approach $1400 \mathrm{GeV}$ and $900 \mathrm{GeV}$ for squark and gluino production, respectively. The grid of letters indicate the channel chosen (a priori, based on best expected limit) to set the limit at the given point in parameter space.

While setting limits on minimal models, such as mSUGRA/CMSSM, can give an indication of the general reach of SUSY searches via colored sparticle production, it is also possible to miss signatures that lie outside the prejudicially constrained parameter space of the minimal models. For example, a simplified model of gluino production cascading to the LSP via decays involving top quarks tends to produce events with many jets but limited $E_{\mathrm{T}}^{\text {miss }}$. To this end, a 'multijet' analysis [5], relying only on jet triggers (thus maintaining sensitivity to more general SUSY scenarios that feature low average $E_{\mathrm{T}}^{\text {miss }}$ ) and requiring between six and nine jets and limited $E_{\mathrm{T}}^{\text {miss }}$, was used to set limits on this model. Again, no significant excess is observed in $4.7 \mathrm{fb}^{-1}$ of data, leading to limits of $m_{\text {gluino }}>820 \mathrm{GeV}$ for any LSP mass of less than $220 \mathrm{GeV}$.

Leptons are a likely product of cascades induced by colored sparticle production that include decays to gauginos and the SUSY partners of heavy quarks. For the $4.7 \mathrm{fb}^{-1}$ dataset, an embellishment of the standard single-lepton-plus-jets analysis involves the inclusion of an $E_{\mathrm{T}}^{\text {miss }}$-triggered soft-lepton channel [6], making use of leptons (e, $\mu$ ) with 


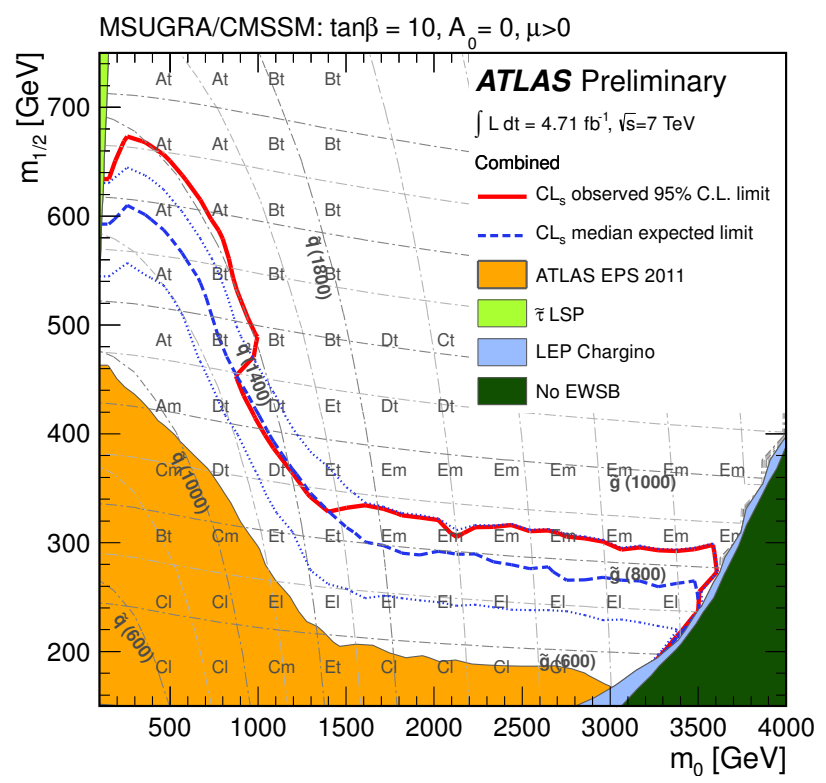

FIGURE 1. Observed and expected exclusion limits in the plane of universal GUT-scale boson $\left(m_{0}\right)$ and

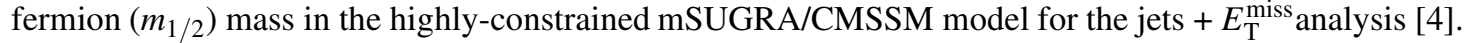
Letters indicate the channel chosen to set the limit at the given point in parameter space.

transverse momenta as low as $7 \mathrm{GeV}$. Interpreted in terms of a phenomenological model involving gluino production followed by a cascade through an intermediate chargino, the failure to observe a significant excess above background in any of the single-leptonplus-jets channels translates into limits of $m_{\text {gluino }}<460 \mathrm{GeV}$ for any LSP mass to within $\sim 10 \mathrm{GeV}$ of the excluded gluino mass (see Fig. 2). The ability to exclude a neutralino LSP with a mass this close to that of the gluino is directly related to the acceptance of soft leptons with $p_{\mathrm{T}}<10 \mathrm{GeV}$.

Searches with photons accompanied by $E_{\mathrm{T}}^{\text {miss }}$ are usually interpreted in terms of GMSB models, for which the possibility (preferred over much of parameter space) of a bino-like NLSP leads to end-of-chain decays into a photon and gravitino, the latter being the source of $E_{\mathrm{T}}^{\text {miss }}$. In $1 \mathrm{fb}^{-1}$ of collision data, no significant excess of events with two identified photons and $E_{\mathrm{T}}^{\text {miss }}>125 \mathrm{GeV}$ is observed. This result is interpreted in terms of a simplified GMSB model for which only the bino-like neutralino NLSP and gluino octet are given accessible masses [7]. Gluinos with mass less than $805 \mathrm{GeV}$ are excluded for any bino mass less than that of the gluino but greater than $50 \mathrm{GeV}$.

SUSY searches with ATLAS data for which all colored sparticles are assumed to be at inaccessibly high masses are discussed elsewhere in these proceedings [8]. In general, for such models, lower limits on the masses of SUSY partners of weakly-interacting SM particles are in the $250-300 \mathrm{GeV}$ range. Additionally, for strong production scenarios for which a third-generation squark dominates the cascade to the LSP - a fairly likely consequence of chiral mixing - multiple-jet final states can degrade the distinctiveness of experimental signatures and thereby degrade mass limits. Analyses geared towards the exploration of such scenarios are discussed in more detail elsewhere in these proceedings [9]; gluino mass limits from such analyses tend to be in the 500-900 GeV range. 


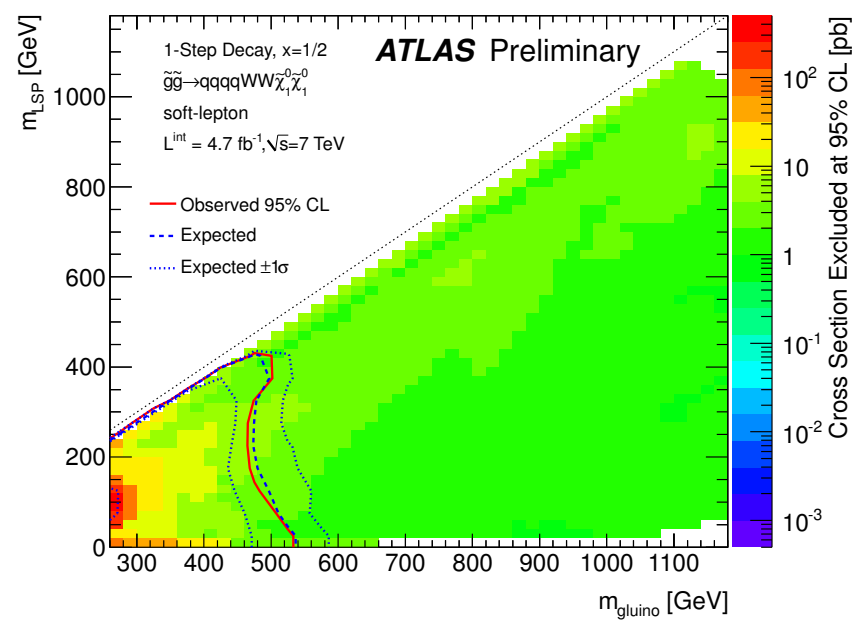

FIGURE 2. Observed and expected limits in the $m_{L S P} / m_{\text {gluino }}$ plane for the $4.7 \mathrm{fb}^{-1}$ single-lepton-plusjets analysis. The ability to exclude neutralino masses close to that of the excluded gluino mass is due to the minimal requirement on the lepton $p_{\mathrm{T}}$.

The near-degeneracy of the wino states $\tilde{\chi}_{1}^{+}$and $\tilde{\chi}_{1}^{0}$ in AMSB can lead to the production of charged metastable particles that decay part-way through the tracking system to the $\tilde{\chi}_{1}^{0}$ LSP and a charged SM particle with momentum too slight to be reconstructed. Such 'disappearing track' signatures have been sought in the number-of-hit distribution of tracks with segments in the highly-redundant Transition Radiation Tracker (TRT) that forms the outer layer of the ATLAS central tracking detector. For the $4.7 \mathrm{fb}^{-1}$ sample, this distribution is statistically identical to that expected for the production of SM particles, leading to a limit of $0.2 \mathrm{~ns}<\tau_{\tilde{\chi}_{1}^{+}}<90 \mathrm{~ns}$ for $m_{\tilde{\chi}_{1}^{+}}<90 \mathrm{GeV}$ [10].

Searches are also performed for several signatures associated with the violation of $R$-parity, which introduces a term [11]

$$
W=-\lambda_{i j k}^{\prime} \tilde{u}_{j} \bar{d}_{k} l_{i}
$$

into the SUSY Lagrangian, which in turn permits a process $d \bar{d} \rightarrow e^{-} \mu^{+}$via $t$-channel top squark ('stop') exchange. In $2.1 \mathrm{fb}^{-1}$ of data, ATLAS has performed the first search for continuum production of a muon and electron of opposite sign [12], finding no excess above the SM expectation. For a coupling parameter product of $\left|\lambda_{131}^{\prime} \lambda_{231}^{\prime}\right|=\left|\lambda_{132}^{\prime} \lambda_{232}^{\prime}\right|=$ 0.05 , such processes are ruled out at $95 \% \mathrm{CL}$ for $m_{\text {stop }}<200 \mathrm{GeV}$.

The BC1 model [13], a more constrained implementation of $R$-parity violation that preserves proton stability, features an unstable stau LSP whose production and decay entails the production of multiple leptons. In $2.1 \mathrm{fb}^{-1}$ of data, no events with greater than three leptons and $E_{\mathrm{T}}^{\text {miss }}>50 \mathrm{GeV}$ [14] are observed, while the SM background is estimated to be $0.7 \pm 0.8$ events. In the context of the $\mathrm{BC} 1$ model this implies limits as high as $1.8 \mathrm{TeV}$ on the gluino mass - the highest limit of any ATLAS SUSY search.

Figure 3 provides a synopsis of ATLAS SUSY search results, including those from a number of analyses not discussed in these proceedings. No discoveries are claimed, and colored sparticle mass limits are generally found to be in the $\mathrm{TeV}$ range, while limits 


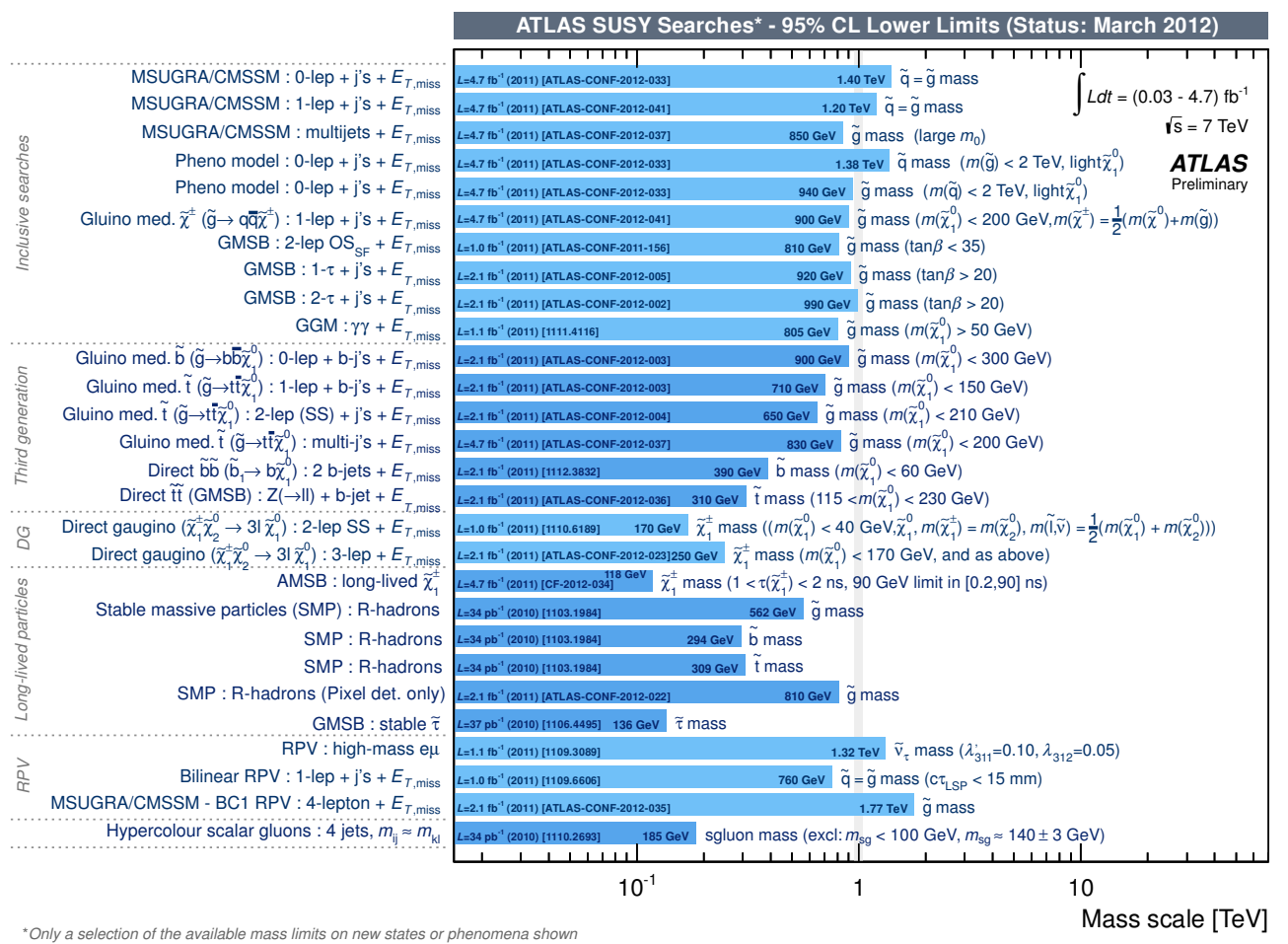

FIGURE 3. Summary of ATLAS SUSY searches as of this conference.

on non-colored sparticle masses remain in the $300-400 \mathrm{GeV}$ range when no colored sparticle mass is accessible at LHC energies. The sensitivity of ATLAS SUSY analyses should increase markedly as higher-luminosity data samples are accumulated at a centerof-mass energy of $8 \mathrm{TeV}$ in 2012, and then as high as $14 \mathrm{TeV}$ in subsequent running.

\section{REFERENCES}

1. A. H. Chamseddine, R. L. Arnowitt, and P. Nath, Phys. Rev. Lett. 49, 970 (1982).

2. M. Dine, W. Fischler, and M. Srednicki, Nucl. Phys. B 189, 575 (1981).

3. L. Randall, and R. Sundrum, Nucl. Phys. B 557, 79 (1999).

4. ATLAS Collaboration, Phys. Lett. B 710, 67 (2012).

5. ATLAS Collaboration, J. High Energy Phys. 11, 99 (2011).

6. ATLAS Collaboration, ATLAS-CONF-2012-041, https://cdsweb.cern.ch/record/1435195.

7. ATLAS Collaboration, Phys. Lett. B 710, 519 (2012).

8. B. Toggerson, "Searches for direct gaugino production and RPV SUSY with leptons at $\sqrt{s}=7 \mathrm{TeV}$ with the ATLAS detector at the LHC," in these proceedings.

9. K. Pajchel, "Search for supersymmetry in events involving third generation squarks and sleptons with ATLAS," in these proceedings.

10. ATLAS Collaboration, Eur. Phys. J. C 72, 1993 (2012).

11. Y. B. Sun et al., Commun. Theor. Phys. 44, 107 (2005).

12. ATLAS Collaboration, Eur. Phys. J. C 72, 2040 (2012).

13. B. Allanach, M. Bernhardt, H. Dreiner, C. Kom, and P. Richardson, Phys. Rev. D 75, 035002 (2007).

14. ATLAS Collaboration, ATLAS-CONF-2012-035, https://cdsweb.cern.ch/record/1432202. 